\title{
Prevention and management of
} musculoskeletal pain in nursing staff by a multifaceted intervention in the workplace: design of a cluster randomized controlled trial with effectiveness, process and economic evaluation (INTEVAL_Spain)

\author{
Consol Serra ${ }^{1,2,3^{*}}$ (D), Mercè Soler-Font ${ }^{1,2}$, Ana María García ${ }^{4}$, Pilar Peña ${ }^{5}$, Sergio Vargas-Prada ${ }^{6,7}$ \\ and José María Ramada 1,2,3
}

\begin{abstract}
Background: Musculoskeletal pain (MSP) is the leading cause of years lived with disability. In consequence, to reduce MSP and its associated sickness absence is a major challenge. Previous interventions have been developed to reduce MSP and improve return to work of workers with MSP, but combined approaches and exhaustive evaluation are needed. The objective of the INTEVAL_Spain project is to evaluate the effectiveness of a multifaceted intervention in the workplace to prevent and manage MSP in nursing staff.

Methods: The study is designed as a two-armed cluster randomized controlled trial with a late intervention control group. The hospital units are the clusters of randomization and participants are nurses and aides. An evidence-based multicomponent intervention was designed combining participatory ergonomics, case management and health promotion. Both the intervention and the control groups receive occupational health care as usual. Data are collected at baseline, and after six and 12 months. The primary outcomes are prevalence of MSP and incidence and duration of sickness absence due to MSP. Secondary outcomes are work role functioning and organizational preventive culture. The intervention process will be assessed through quantitative indicators of recruitment, context, reach, dose supplied, dose received, fidelity and satisfaction, and qualitative approaches including discussion groups of participants and experts. The economic evaluation will include cost-effectiveness and cost-utility, calculated from the societal and the National Health System perspectives.

Discussion: Workplace health programs are one of the best options for the prevention and control of non-communicable diseases. The main feature of this study is its multifaceted, multidisciplinary and de-medicalized intervention, which encompasses three evidence-based interventions and covers all three levels of prevention, which have not been previously unified in a single intervention. Also, it includes a comprehensive quantitative and qualitative evaluation of the intervention process, health results, and economic impact. This study could open the possibility of a new paradigm for the prevention and management of MSP and associated sickness absence approach at the workplace.
\end{abstract}

(Continued on next page)

\footnotetext{
* Correspondence: consol.serra@upf.edu

${ }^{1}$ Centre for Research in Occupational Health, Pompeu Fabra University/

IMIM-Hospital del Mar Medical Research Institute, PRBB-Barcelona Biomedical

Research Park. Dr. Aiguader, 88, 08003 Barcelona, Spain

${ }^{2}$ CIBER of Epidemiology and Public Health, Barcelona, Spain

Full list of author information is available at the end of the article
}

(c) The Author(s). 2019 Open Access This article is distributed under the terms of the Creative Commons Attribution 4.0 International License (http://creativecommons.org/licenses/by/4.0/), which permits unrestricted use, distribution, and reproduction in any medium, provided you give appropriate credit to the original author(s) and the source, provide a link to the Creative Commons license, and indicate if changes were made. The Creative Commons Public Domain Dedication waiver (http://creativecommons.org/publicdomain/zero/1.0/) applies to the data made available in this article, unless otherwise stated. 
(Continued from previous page)

Trial registration: Current Controlled Trials ISRCTN15780649 Retrospectively registered 13th July 2018.

Keywords: Musculoskeletal pain, Return to work, Cluster randomized controlled trial, Sick leave, Multifaceted intervention, Ergonomics, Case management, Health promotion

\section{Background}

Musculoskeletal pain (MSP) and associated limitations in mobility and functional capacity are essential characteristics of musculoskeletal disorders (MSDs) [1]. Upper extremity and low back are the most common pain locations. During the last two decades low back pain has been the leading cause of years lived with disability, and its global prevalence and incidence still show an increasing trend [2]. Healthcare workers are an occupational group at high risk of developing MSP [3-5].

In Europe, there is evidence that around $70-80 \%$ of workers report discomfort due to awkward postures and forceful work [2,3] with an impact not only on health but also on work and economy, representing $50 \%$ of sickness absence episodes and $60 \%$ of permanent disabilities [6]. This scenario generates new challenges on health systems, requiring new strategies for its prevention and management $[7,8]$.

International variations of the distribution of MSP in the population support that its onset and persistence are influenced by a complex and dynamic interaction between biological, psychosocial, cultural and also individual factors [9-11]. The biopsychosocial model simultaneously considers all these factors and their impact on health and general well-being [12]. Although this model has become the dominant framework through which the etiology and prognosis of MSDs is conceptualized, its application into practice has not been optimal [13]. Traditionally, the causes of MSDs, and also MSP, have been investigated through biomechanics, physiology, genetics, epidemiology and rehabilitation, but separated from other involved relevant disciplines [14]. This fragmented approach does not offer optimal management. Evidence suggests that a combination of several specific approaches in multi-component interventions that address various determinants of the problem and those that incorporate a workers' participatory approach in the intervention process can obtain better results [15-17].

The INTEVAL_Spain is an evidence-based intervention and consists of three components: participatory ergonomics (primary prevention of occupational risk factors), case management (secondary and tertiary prevention) and promotion of healthy lifestyles at work. There is an increasing concern on the so-called participatory ergonomics programs because they are both multi-factorial and participatory [18] and are defined as interventions in the workplace in which the relevant company agents actively participate with the aims to identify and act over the determinants of MSDs [19, 20]. Participatory ergonomics programs have been tested in Spanish companies with promising results [21, 22]. There is scientific evidence that the management of musculoskeletal symptoms through a tailored approach could be more efficient if individuals are stratified according to certain prognostic profiles in the initial stages of the disorder and after treatment [23, 24]. At tertiary prevention, the available scientific evidence suggests that case management can reduce the duration of sickness absence, musculoskeletal symptoms and disability, and improve work continuity [25-27]. Integrating activities on healthy lifestyles at work is also an important component of occupational health programs to reduce MSP [28], so different strategies promoting workers' physical activity, emotional wellbeing and healthy diet [29-32] were also included. As for healthcare workers, promoting healthy lifestyles should be a priority because of its double impact on their own health and that of patients, encouraging these lifestyles within the general population. In addition, the World Health Organization considers workplace as one of the best contexts for the prevention and control of non-communicable diseases [33].

This paper presents the design of a study that evaluates the multifaceted INTEVAL_Spain intervention in the workplace in nursing staff from two tertiary hospitals to prevent and manage MSP and its associated sickness absence. We hypothesize that this intervention will be effective in terms of reducing the prevalence of MSP and will also reduce the incidence of associated sickness absence and the time to return to work in the intervention group at the end of the study, compared to the control non-intervention group. In addition, a process evaluation of the intervention and its association with the impact on MSP, and an economic evaluation will be carried out.

\section{Methods}

The CONSORT statement and the extension for cluster randomized trials were used to describe the design of the study $[34,35]$.

\section{Study design and context}

The study is designed as a two-armed cluster randomized trial with a late intervention control group (Fig. 1) where clusters are independent hospital units, and 


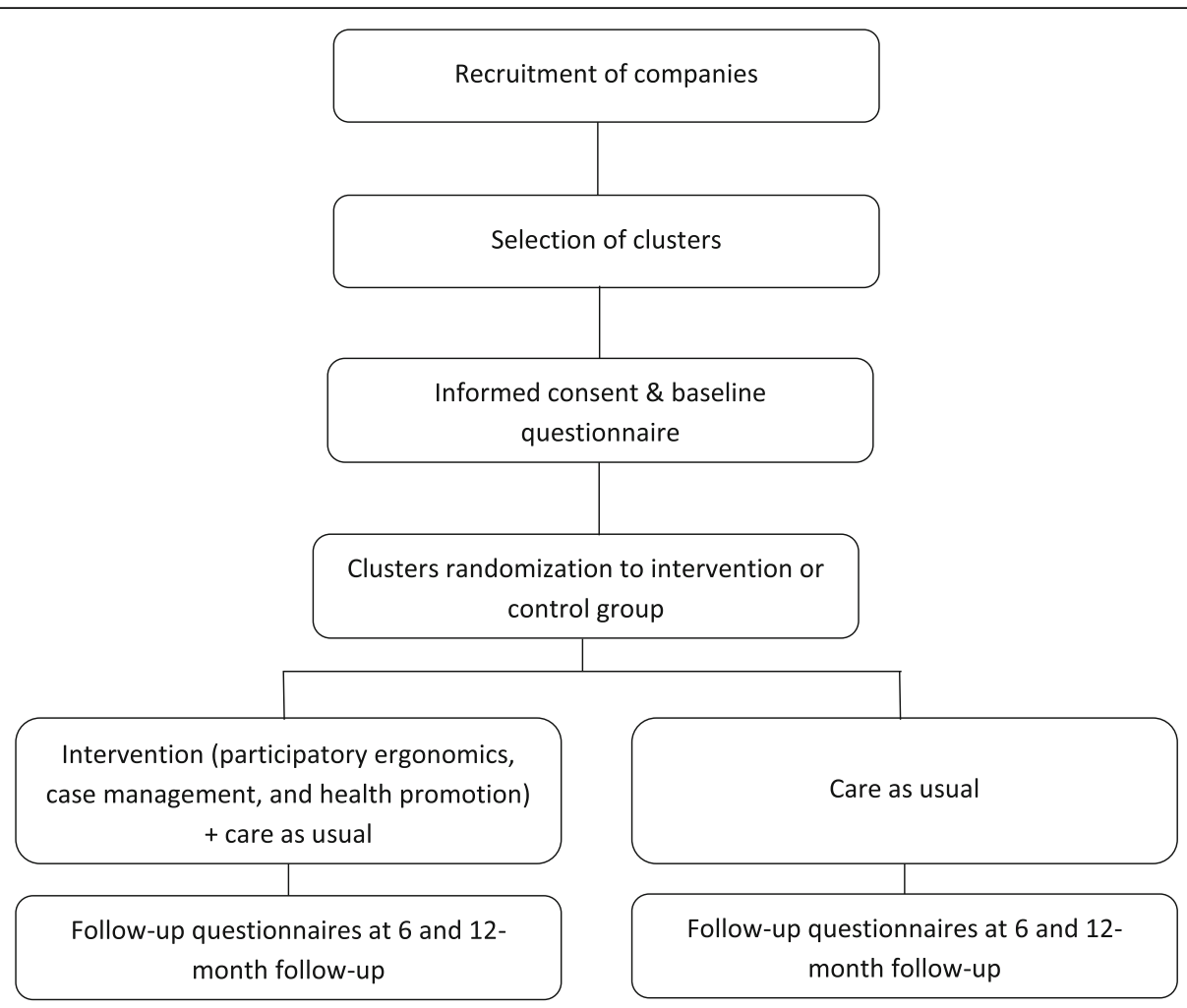

Fig. 1 Flowchart and overview of the trial

participants are nursing staff (nurses and aides) highly exposed to ergonomic risk factors at work. There is extensive scientific evidence that incidence and prevalence of MSP is very high and is the leading cause of sickness absence in healthcare workers, especially nurses and aides working in hospitals [36], as it is exposure to ergonomic and other risk factors [37, 38]. It is also well known that other non-occupational, cultural and individual factors also play an important role in the incidence and prognosis of MSP [36]. In Spain, as in other European countries, all employers are required to organize some kind of occupational health service (OHS) according to exposure to occupational risks and company size. The tasks of these OHS include traditional risk assessment and investigation of occupational injuries, health surveillance mainly through health examinations, prevention, training and information covering occupational and non-occupational health risks. External OHS are usually the main occupational health provider for small and medium size companies, whereas large companies, including hospitals, usually have an inhouse OHS which offer better opportunities for research and testing new approaches to improve workers'health.

The study is conducted in two main tertiary hospitals in the cities of Barcelona and Sabadell (Barcelona province) with similar level of health care complexity, a workforce of around 4000 workers each, of whom around $60 \%$ are nursing staff, and with an experienced in-house OHS each, that share common methodologies and good coordination.

\section{Recruitment of companies}

Hospitals had to meet the following criteria to guarantee the selection of appropriate clusters and the implementation of the intervention: (1) workforce of at least 500 workers, (2) commitment and explicit interest from the hospital management to carry out the intervention and its evaluation; (3) existence of an in-house OHS that maintains routine quality records and is interested in developing the intervention and; (4) existence of work units with exposure to significant musculoskeletal risks, as assessed by the OHS.

Based on these criteria, two tertiary hospitals with specialized acute care, psychiatry, long-term and primary care were selected.

\section{Recruitment of participants}

To recruit participants, informative sessions were performed at each cluster before disclosing whether they would be allocated to the intervention or control group, and informed consent and the baseline questionnaires were obtained from each participant worker. After these documents were filled in and returned, the units were 
randomized and informed about their condition of being intervention or control group, and the intervention started. Follow-up questionnaires are administered at six and 12 months. The questionnaires are anonymous to promote participation and worker's confidence towards the intervention. Anonymized data related to sickness absence are collected for the period from 1 year before the intervention until the end of the study.

\section{Eligibility criteria}

The inclusion criteria are nursing staff (nurses and aides), including employees on sickness absence, who voluntarily accept to participate. Workers with a temporal contract during a short period, working in several units and on sabbatical leave are excluded.

\section{Intervention}

The intervention covers the three levels of prevention (primary, secondary and tertiary prevention), lasts for 1 year and is implemented to the control group thereafter.

For primary prevention of occupational risk factors, a standardized procedure of participatory ergonomics named ERGOPAR, developed and previously piloted in Spain is used [39]. A working group (ERGO group) is organized for each hospital unit, including a specialist in ergonomics, the unit supervisor/s, one referent worker for each shift (morning, afternoon and two night shifts) and one workers' union representative. This ERGO group is responsible for the development and implementation of the intervention, and receive basic training in ergonomics and participatory methods. The intervention begins with the diagnostic phase, with the distribution of a previously validated self-completed questionnaire in which data on MSP and exposure to musculoskeletal risk factors at work are collected using specific risk assessment. This information is then analyzed and discussed by the ERGO group and a prioritized list of risk factors is developed. The next step is the treatment phase, in which the information collected in the questionnaires is shared and discussed with coworkers in the so-called prevention circles. These circles propose a prioritized list of preventive measures aimed to avoid or reduce the identified ergonomic risk factors. This list may include structural, technical, organizational, training and/or information measures for improvement, and workplace modifications. The OHS coordinates the implementation of these measures along with the corresponding department managers. Based on the previous available experience with the ERGOPAR method [22], the time required to complete all phases of the intervention in each work unit has been estimated from six to 12 months.

Secondary and tertiary prevention is carried out through a tailored case management intervention.
Participant workers are voluntarily referred to case management either by themselves, by proposal of their supervisor or a physician of the OHS. Workers with a serious underlying organic pathology are excluded from this intervention component and managed according to standard medical practice. A trained case manager assigns participants to three strata of management and treatment, according to their level of risk for persistent musculoskeletal symptoms: low, medium or high. This profile is obtained by telephone interview using a questionnaire made of validated tools to generate a risk profile and that assesses the presence of radiated pain, comorbidity, limitations to carry out daily activities, discomfort derived from pain, fear of movement, beliefs and negative expectations regarding the prognosis of pain, the presence of anxiety and of other mood disorders [23, 24, 38, 40-43]. Workers assigned to the low-risk group attend an education session on health beliefs related to MSP. Workers assigned to the medium and high-risk groups receive specific and tailored treatment including rehabilitation, physiotherapy and cognitive-behavioral therapy. In parallel, cases may be discussed at the weekly clinical session with members of the OHS to evaluate possible specific needs at work, as job adjustments or improvements to help workers to stay and/or early return to work. The case manager contacts all workers regularly to carry out a motivational telephone follow-up, and also coordinates the services and plan the sessions.

An evidence-based program to promote healthy lifestyles at work is also part of the intervention. This program is addressed and offered to all nurses and aides of the participating intervention units, and application is voluntary and free. It includes: (1) mindfulness training, defined as a self-regulation approach to stress reduction and emotional management [44], consisting of an adapted course of 4 sessions of $2 \mathrm{~h}$ each on MBRS (Mindfulness-based Stress Reduction) training which has been shown to be effective in healthcare workers [29]; (2) Nordic Walking training, defined as a walking technique that uses specially designed poles to actively involve the upper body and arms with wide scientific evidence of its benefits on various health outcomes, including MSP and MSDs [30], offering a program of 12 sessions of $1.5 \mathrm{~h} /$ session during 12 weeks; and (3) healthy eating based on the Mediterranean diet, as one of the healthiest dietary models that currently exist $[31,32]$ consisting on a $3 \mathrm{~h}$ session and a web platform.

Finally, all components of the intervention are integrated and require coordination by a champion who acts as a leader and facilitator of the intervention, organising and leading the work to be developed by the study team. His/her tasks involve communication, meetings planning (i.e. informative sessions, and research team meetings), 
organization of the health promotion activities (i.e. calendar planning), collection and data processing and writing of reports.

\section{Occupational health care as usual}

During the intervention period, the OHS of each participating hospital keeps providing the standard occupational health practices for both the intervention and control units. These practices include usual occupational risk assessments, investigation of occupational injuries, health surveillance, smoking cessation, training, information and expert assessment in occupational health at all levels in the hospital (i.e. managers, supervisors, workers), as well as the usual support program for return to work mainly focused on interventions related to workplace adaptations, clinical support and management of permanent disability.

\section{Measurements and procedure}

Data are collected by standardized, validated questionnaires, processed in registers and a sample was double-checked to identify inconsistencies and errors. Self-reported questionnaires are administered at baseline, six and 12-month follow-up. Details on process evaluation are also stored in standardized registers, and double-checked. Data of sickness absence will be extracted from the company registries.

\section{Primary outcomes}

\section{Prevalence of musculoskeletal self-perceived pain}

The Spanish adaptation of the Nordic Questionnaire from the ERGOPAR Method [45] is used to measure self-perceived MSP in the neck, shoulders and upper back, low back, elbows, hands, legs, knees, and feet, through the dichotomous question "Do you have discomfort or pain in this area?". Data are collected at baseline, six and 12-month follow-up.

\section{Sickness absence}

Data on episodes and duration of sickness absence due to a musculoskeletal condition (MSP or MSDs) are collected from the company registries and the OHS during the study period and 1 year before the intervention.

\section{Secondary outcomes Work functioning}

Work functioning is measured at baseline, six and 12-month follow-up using the Work Role Functioning Questionnaire-Spanish Version (WRFQ-SpV) [46-48]. This tool is a self-administered questionnaire that measures perceived difficulties in performing one's job due to health problems [49] and consists of 27 items divided into five subdomains: work scheduling demands, output demands, physical demands, mental demands, and social demands. The score of this questionnaire ranges from 0 to 100 , being 100 the maximum score (having $100 \%$ of your functional capacity).

\section{Organizational preventive culture}

Organizational preventive culture is measured by the Institute for Work \& Health Organizational Performance Metric (IWH-OPM) [50]. The IWH-OPM is an evidence-based, eight-item questionnaire used to help organizations assess and improve their health and safety performance and is measured at baseline, six and 12-month follow up.

\section{Process evaluation}

The intervention process will be evaluated based on previous evidence and indicators for process evaluation [51, 52]: recruitment, context, reach, dose delivered, dose received, fidelity and satisfaction. Additionally, the stakeholder's role will also be included (implementation strategy). Process evaluation data will be collected by means of questionnaires, the champion registries and qualitative approaches (discussion groups with researchers and participants). Recruitment, context and reach indicators will be available in the intervention and control group; dose delivered, dose received, fidelity and satisfaction will only be available for the intervention group since these indicators refer to various aspects of the intervention itself.

Context information will be collected through discussion groups and also, at six and 12-month follow-up with three questions related to the aspects that affect their usual workload (improvement in the manual mobilization of patients, technical aids, and load handling). Recruitment, reach, dose delivered and dose received, and fidelity (of the intervention and adherence of the participants) data will be extracted from the champion registers. Recruitment refers to the procedures used to approach and attract prospective program participants, as defined as the proportion of possible workers who agreed to participate in the study signing the informed consent at baseline. Reach can be defined as the proportion of the intended target audience that participates in an intervention, according to the intervention and control group reach and will be calculated considering the proportion of people who answered the questionnaires from those who signed the informed consent. For each component of the intervention, reach will be calculated as the proportion of people who have participated in each one. Dose delivered will be calculated as the number of hours of offered services, and dose received as the extent to which participants have actively participated in each component of the intervention. The intervention and participants fidelity will also be assessed. We operatized the fidelity of the intervention 
as a ratio between the planned and the developed one, and the fidelity of the participants as adherence through the proportion between their attendance and the dose delivered. Questions on satisfaction are self-administered at the end of each component of the intervention and at the 12-month follow-up questionnaire, through the questions "Have you met your expectations?" and "in general, what is your satisfaction?" on a scale of 1 to 10 , being 10 the maximum satisfaction. Qualitative data from the discussion groups will be used to identify the key points and the possible improvements, as the implementation strategy indicator.

\section{Economic evaluation}

A cost-utility and cost-effectiveness analysis will be performed from the societal and the National Health System perspectives. The cost-utility analysis will be conducted to analyze changes in quality-adjusted life years (QALYs) measured by EQ-5D-3 L [41], and the corresponding costs for each perspective. Moreover, the cost-effectiveness analysis will compare changes in MSP to the costs for each perspective. The national health system perspective will include the direct costs of the Spanish public health services (direct costs of the disease: visits to the GP, specialists, diagnostic tests and medication) and the costs of the intervention; the societal perspective will include all these costs and also the loss of production (indirect costs). Direct costs will be estimated based on administrative data from the clinical registers. Indirect costs will be estimated using the human capital approach from sick leave (company registries). Costs of the intervention (i.e. time of experts) will be obtained from the study register and will become unit costs according to the corresponding collective agreements.

\section{Randomization}

Nursing staff (nurses and aides) of the hospital units with higher exposure to musculoskeletal risk due to ergonomic risk factors at the hospital unit and the type of patients (medium and high dependency) are selected. An independent researcher assigns the clusters to the intervention group or to the control group by simple randomization stratified by center.

\section{Blinding}

In this cluster randomized trial blinding was not possible. The condition of being included in the intervention or the control group cannot be blinded but the clusters are randomized after signing informed consent and completing the baseline questionnaire. The services provided and the participating OHS professionals cannot be blinded because they are involved in the implementation of the intervention.

\section{Data analysis}

Statistical analyses adapted for cluster-randomized controlled trials will be conducted. Descriptive analyses of the participants' characteristics and comparisons between the intervention and control clusters will be performed. The generalized estimation equations (GEE) procedure will be used for the analysis of the MSP, work functioning and organizational preventive culture comparing the difference from the baseline to the 12-month follow-up of the intervention group, with the difference from the baseline to the 12-month follow-up of the control group. The models will be adjusted by the cluster design and by the potential confounders. The incidence of sickness absence will be analyzed through a logistic regression model, taking as a reference the episodes started the year before the intervention and the control group. A Cox proportional hazard model will estimate the hazard ratio of returning to work earlier after the intervention in the intervention group compared to the control group.

Statistical analyses will be performed with STATA 13 (StataCorp, 2013. Stata Statistical Software: Release 13. College Station, TX: StataCorp LP).

\section{Statistical power}

The sample size estimation is based on the prevalence MSP estimated at $80 \%$ for healthcare workers [3], the impact of the intervention expecting a reduction of a $20 \%$ in the prevalence of musculoskeletal pain [53, 54], alpha values (type I error) $=0.05$, statistical power $=0.80$ and interclass correlation coefficient $(\mathrm{ICC})=0.05$. Applying these criteria, we obtained a minimum sample of 164 subjects, 82 in each group. The units of the participating hospitals have a varied number of workers (20 to $60)$. Taking into account the sample size calculations, 8 units have been randomized.

\section{Discussion}

A multifaceted intervention on MSP in hospital nursing and aide staff has been designed and described. The impact on MSP in the population shows that this is one of the main health challenges that must be addressed in Spain and similar countries in terms of health, work and economy $[2,3,6]$.

\section{Methodological considerations}

The randomized controlled trial (RCT) is the basic methodological paradigm for the evaluation of health interventions. Randomization guarantees that the assignment of a work unit to the intervention or control group is exclusively due to chance, thus avoiding effects of confounding and selection biases. The availability of a control group makes it possible to distinguish between 
epidemiological and/or statistical associations and cause-effect relations.

The INTEVAL_Spain has some strengths that make it unique in occupational health. First, it is a multifaceted intervention that includes the three preventive levels (primary, secondary, tertiary) simultaneously to reduce MSP in workers. Secondly, it has a multidisciplinary, de-medicalized and participatory approach, as it requires the involvement of the main agents in the company (managers, workers, technicians). And third, this study places special emphasis on the evaluation of results, process, and economics, and uses quantitative and qualitative methods for collecting data to achieve a comprehensive and accurate assessment at all levels of the intervention. In addition, this study places special emphasis on the evaluation of results, process and economics, and consists of mixed methods of collecting data (qualitative and quantitative) to achieve a comprehensive and accurate assessment at all levels of the intervention.

Our study has also potential limitations that we need to consider. There may be a participation bias, since workers with MSP could be more interested in participating, and otherwise, workers with good health could not feel the need of participating. Therefore, a sensitivity analysis comparing the sociodemographic variables of the participants and non-participants of each cluster will be carried out in order to quantify it. Moreover, there could be contamination, which could result in an underestimation of the effectiveness. However, clusters are different units located in different buildings and/or different floors, and therefore may not have much contact between them. Also, as it is a specific population (nurses and aides of public hospitals), the external validity of the results concerning the working population may be limited. Finally, questionnaires are anonymous and an identifier is not available. The research team made this decision to encourage participation and make sure that participants were feeling comfortable with the study, since the questionnaires were self-completed in the workplace and included some personal questions, as well as questions about the relationship with the supervisors. However, we are aware that this decision could limit the statistical power and entail an underestimation of the results.

\section{Possible impact of results}

The preventive intervention evaluated in the INTEVAL_Spain project is characterized by its flexibility, efficiency and capacity to adapt to different companies' needs. In addition, it is designed to optimize and make most of existing prevention resources in companies cost-effective. The whole project, in fact, is based on the sum of capacities of the different participants: managers, workers and their representatives, researchers, and occupational health specialists, taking advantage of the strengths and potentials of each of these agents and is carried out in close collaboration between the participating companies. This condition is both an opportunity and a challenge. An opportunity, since the direct interaction with the company's agents in the development of the research strengthens the relationships among research centers, researchers themselves and the company, and facilitates the transfer of results to their direct users and targets. It is also a common challenge in occupational health intervention studies, as it is necessary to achieve the adjustment between the methodological requirements of the research and times, expectations and needs of the productive activity in the company.

This study aims to promote a change of orientation and a new paradigm for the prevention and management of MSP and associated sickness absence. The idea of implementing these combined and non-medicalized interventions in the OHS in a sustainable way over time could facilitate access for an early management of MSP at work, improve their health and be cost-effective.

\section{Abbreviations}

MSDs: Musculoskeletal disorders; MSP: Musculoskeletal pain; OHS: Occupational Health Service

\section{Acknowledgements}

We want to thank all healthcare workers and their representatives, referents, managers and supervisors from the hospital clusters of Parc de Salut Mar (PSMar) and Corporació Sanitaria Parc Taulí (CSPT) who agreed to participate in the trial. Especially, PSMar: Pilar Pastor (Ward manager), Isabel Aranega, Noemí Cajete, Raúl Martín, Dolores Rincón, Nuria Saavedra (UH30); Rosa Balaguer (ward manager), Sonia Advíncula, Nuria Esteban, Montse Regordosa, Cristina Salvat, Ana Uribe (Intensive Care Unit); Isabel Egea (ward manager), Ana Delgado, M Ángeles Fernández, Josefa García, Susana Margalef, Alexandra Morales, Ana M Rodríguez, Isabel Rodríguez (Llevants 3,4); Montse Sitges, Txell Gumà (ward managers), Rosa Elias, Lucía Fernández, Ana M Luque, Nuria Morillas, Carlos Perez, Sandra Vives (Surgical area); Elena Maull, Desirée Ruiz (ward manager), Alberto Gonzalez, Antonia Rincón, Bernat Sarrió, Gina Shakya (UH04); Beatriz Fernández (ward manager), Ma Encarnación Avilés, Miriam Hernández, Naza Martinez, Carme Pellín, Nenita de los Santos, Sergio Taibo, Chari Villanueva (Acute geriatrics unit). Rosa Aceña, Cuca Esperanza and Núria Pujolar (Nursing Coordinators); Mercedes Calvo, Miguel Celada, Lluisa Cosp, Eugenio Gurrea, Montse Sallés, Pilar Serrano (Nurse Supervisors); and Vicky Abad, Pilar González, Francisco Martos (Prevention Delegates). CSPT: Isabel Simó (ward manager), Ma Goretti Gelonch, Elisabeth Mérida, Sara Purcalla, Mónica Sianes (UH06); José Ma Barradas (ward manager), Judith Camps, M José González, Verònica Gómez, Victoria Plaza, Estefanía del Pino (UH08); and Elena Polo (Prevention Delegate)

We also want to thank the contribution of Toni Merelles (University of Valencia) for taking the lead of the funding coordination, Chelo Sancho (specialist in participatory ergonomics), Rocío Villar (occupational physician, PSMar); Victòria Lopez (occupational nurse, CSPT); Cristina Cervantes, Ferran Escalada and the physiotherapists team (Rehabilitation Service, PSMar);

Fernanda Caballero and physiotherapists team (Rehabilitation Service, CSPT); Gemma Salvador (Agència de Salut Pública de Catalunya) and Ada Parellada (Chef); Anna Amat (champion and case manager), Carmen de la Flor (champion), Montserrat Fernandez (CiSAL, UPF); Cristina Giménez (Psychologist); Antonio Brieba (Nordic walking instructor); Georgina Badosa and Mónica Astals (Mindfulness instructors).

We would like to acknowledge Mireya Jaramillo for providing a grammatical revision of the text. 


\section{Funding}

The study is funded by the Instituto de Salud Carlos III-FEDER (reference numbers PI14/01959, December 2014; and PI17/00779, October 2017) and Fundación Prevent (December 2016). The funders had no role in the study design, data collection and analysis, decision to publish, or preparation of the manuscript.

\section{Availability of data and materials}

Data sharing is limited to the study research team and all analyses will be carried out internally.

\section{Authors' contributions}

CS is the current project leader, co-author of the original version of the protocol for the provision of funding, is the field work coordinator of Parc de Salut Mar (Barcelona) and co-drafted the manuscript. MS contributed to the study design and measurements, and drafted the first version of this manuscript. AMG wrote the original version of the protocol for the provision of funding as a project leader, and contributed to the manuscript. PP is the field work coordinator of Corporació Parc Taulí (Sabadell), participated in the design and implementation of the study and contributed to the manuscript. SVP randomized the clusters, contributed to the planning of different scales for the diverse outcome measurements, and contributed to the manuscript. JMR participated in the design, planning and implementation of the study, and contributed to the manuscript. All authors have read and approved the final version.

\section{Ethics approval and consent to participate}

Ethical approval was obtained from the Clinical Research Ethical Committee of the Parc de Salut Mar, reference number: 2014/5714/1. Informed written consent is obtained from all participants. The written and oral information that given to the participants, stresses that their participation is voluntary and that they may withdraw at any time without having to give a reason.

\section{Consent for publication}

Not applicable.

\section{Competing interests}

The authors declare that they have no competing interests.

\section{Publisher's Note}

Springer Nature remains neutral with regard to jurisdictional claims in published maps and institutional affiliations.

\section{Author details}

${ }^{1}$ Centre for Research in Occupational Health, Pompeu Fabra University/ IMIM-Hospital del Mar Medical Research Institute, PRBB-Barcelona Biomedical Research Park. Dr. Aiguader, 88, 08003 Barcelona, Spain. ${ }^{2}$ CIBER of Epidemiology and Public Health, Barcelona, Spain. ${ }^{3}$ Occupational Health Service, Parc de Salut Mar, Barcelona, Spain. ${ }^{4}$ Department of Public Health, University of Valencia, Valencia, Spain. ${ }^{5}$ Occupational Health Service, Corporació Sanitària Parc Taulí, Sabadell, Spain. ${ }^{6}$ Healthy Working Lives Group, Institute of Health and Wellbeing, College of Medical, Veterinary and Life Sciences, University of Glasgow, Glasgow, UK. ${ }^{7}$ Salus Occupational Health, Safety and Return to Work Services, NHS Lanarkshire, Hamilton, UK.

\section{Received: 14 December 2018 Accepted: 20 March 2019} Published online: 28 March 2019

\section{References}

1. World Health Organization. Occupational health. Musculoskeletal conditions. WHO. 2018. http://www.who.int/mediacentre/factsheets/musculoskeletal/en. Accessed 1 May 2018.

2. GBD 2016. Disease and Injury Incidence and Prevalence Collaborators. Lancet. 2017;390:1211-59.

3. Pinilla García J, Almodóvar Molina A, Galiana Blanco ML, Hervás Rivero P, Zimmermann Verdejo M. Encuesta Nacional de Condiciones de Trabajo. 2015 6a EWCS - España. Madrid (Spain): Instituto Nacional de Seguridad e Higiene en el Trabajo; 2017.

4. Holtermann A, Clausen T, Aust B, Mortensen OS, Andersen LL. Risk for low back pain from diferent frequencies, load mass and trunk postures of lifting and carrying among female healthcare workers. Int Arch Occup Environ Health. 2013;86-4:463-70.

5. Wang S, Liu L, Lu M, Koo M. Comparisons of musculoskeletal disorders among ten different medical professions in Taiwan: a nationwide, population-based study. PLos One. 2015;10-4:1-10.

6. Okunribido O, Wynn T. Aging and work-related musculoskeletal disorders: a review of the recent literature. Norwich: Health and Safety Executive; 2010. p. RR799.

7. Murray CJL, Vos T, Lozano R, et al. Disability-adjusted life years (DALYS) for 291 diseases and injuries in 21 regions, 1990-2010: a systematic analysis for the global burden of disease study 2010. Lancet. 2012;380:2197-223.

8. Vos T, Flaxman AD, Naghavi M, Lozano R, Michaud C, Ezzati M, et al. Years lived with disability (YLDs) for 1160 squealed of 289 diseases and injuries 1990-2010: a systematic analysis for the Global Burden of Disease Study 2010. Lancet. 2012;380-9859:2163-96.

9. National Research Council. Steering Committee for the Workshop on WorkRelated Musculoskeletal Injuries. Work-Related Musculoskeletal Disorders: Report, Workshop Summary, and Workshop Papers. Washington: National Academy Press; 1999.

10. Coggon D, Ntani G, Vargas-Prada S, Martinez JM, Serra C, Benavides FG, Palmer KT. Members of CUPID Collaboration International variation in absence from work attributed to musculoskeletal illness: findings from the CUPID study. Occup Environ Med. 2013;70:575-84.

11. Bernal D, Campos-Serna J, Tobias A, Vargas-Prada S, Benavides FG, Serra C. Work-related psychosocial risk factors and musculoskeletal disorders in hospital nurses and nursing aides: A systematic review and meta-analysis. Int J Nurs Stud. 2015;52:635-48.

12. Engel $\mathrm{G}$. The need for a new medical model: a challenge for biomedicine Science. 1977;196-4286:129-36.

13. Carnes D, Homer K, Underwood M, Pincus T, Rahman A, Taylor S. Pain management for chronic musculoskeletal conditions: the development of an evidence-based and theory-informed pain self-management course. BMJ Open. 2013;3-11:e003534.

14. Marras WS. State-of-the-art research perspectives on musculoskeletal disorder causation and control: the need for an intergraded understanding of risk. J Electromyogr Kinesiol. 2004;14-1:1-5.

15. Hoefsmit N, Houkes I, Nijhuis FJ. Intervention characteristics that facilitate return to work after sickness absence: a systematic literature review. J Occup Rehabil. 2012;22-4:462-77.

16. Cullen KL, Irvin E, Collie A, Clay F, Gensby U, Jennings PA, et al. Effectiveness of workplace interventions in return-to-work for musculoskeletal, painrelated and mental health conditions: an update of the evidence and messages for practitioners. J Occup Rehabil. 2018;28-1:1-15.

17. Rasmussen CDN, Holtermann A, Bay H, Sogaard K, Jorgensen MB. A multifaceted workplace intervention for low back pain in nurses' aides: a pragmatic stepped wedge cluster randomised controlled trial. Pain. 2015;156:1786-94.

18. Silverstein B, Clark R. Interventions to reduce work-related musculoskeletal disorders. J Electromyogr Kinesiol. 2004;14:135-52.

19. Van Eerd D, Cole D, Irvin E, Mahood Q, Keown K, Theberge N, et al. Process and implementation of participatory ergonomic interventions: a systematic review. Ergonomics. 2010;53-10:1153-66.

20. García AM, Gadea R, Sevilla MJ, Genís S, Ronda E. Participatory ergonomics: a model for the prevention of occupational musculoskeletal disorders. Rev Esp Salud Pública. 2009;83:509-18.

21. García AM, Sevilla MJ, Gadea R, Casañ C. Intervención de ergonomía participativa en una empresa del sector químico. Gac Sanit. 2012;26-4:383-6.

22. García AM, Boix P, Benavides FG, Gadea R, Rodrigo F, Serra C. Participación para mejorar las condiciones de trabajo: evidencias y experiencias. Gac Sanit. 2016;30-S1:87-92.

23. Hill JC, Dunn KM, Lewis M, Mullis R, Main CJ, Foster NE, Hay EM. A Primary Care Back Pain Screening Tool: Identifying Patient Subgroups for Initial Treatment. Arthritis Rheum. 2008;59-5:632-41.

24. Hill JC, Whitehurst DGT, Lewis M, Bryan S, Dunn KM, Foster NE, et al. Comparison of stratified primary care management for low Back pain with current best practice (STarT Back): a randomized controlled trial. Lancet. 2011;378:1560-71.

25. Demou E, Gibson I, Macdonald EB. Identification of the factors associated with outcomes in a condition management Programme. BMC Public Health. 2012;12:927.

26. Kamper SJ, Apeldoorn AT, Chiarotto A, Smeets RJ, Ostelo RW, Guzman J, et al. Multidisciplinary biopsychosocial rehabilitation for chronic low back pain. Cochrane Database Syst Rev. 2014;9:CD000963. 
27. Brown J, Mackay D, Demou E, Craig J, Frank J, Macdonald EB. The EASY (Early Access to Support for You) sickness absence service: a four-year evaluation of the impact on absenteeism. Scand J Work Environ Health. 2015:41-2:204-15.

28. The National Institute of Occupational Safety and Health (NIOSH). NIOSH Total Worker Health. Webinar Series. https://www.cdc.gov/niosh/twh/. Accessed 21 Mar 2018

29. Zeller JM, Levin PF. Mindfulness interventions to reduce stress among nursing personnel: An occupational health perspective. Workplace Health Saf. 2013;61-2:85-9.

30. Tschentscher M, Niederseer D, Niebauer J. Health benefits of Nordic Walking: a systematic review. Am J Prev Med. 2013;44-1:76-84.

31. Martínez-González MA, Corella D, Salas-Salvadó J, Ros E, Covas MI, Fiol M et al. Cohort Profile: design and methods of the PREDIMED study. Int J Epidemiol. 2012:41-2:377-85.

32. Saulle R, Semyonov L, La Torre G. Cost and cost-effectiveness of the Mediterranean diet: results of a systematic review. Nutrients. 2013;5-11: 4566-86.

33. World Health Organization. Occupational health. Workplace health promotion. 2018. http://www.who.int/occupational_health/topics/ workplace/en/. Accessed 1 Oct 2018.

34. Altman DG, Schulz KF, Moher D, Egger M, Davidoff F, Elbourne D, Gotzsche PC, Lang T. The revised CONSORT statement for reporting randomized trials: explanation and elaboration. Ann Intern Med. 2001;134:663-94.

35. Campbell MK, Piaggio G, Elbourne DR, Altman DG. Consort 2010 statement: extension to cluster randomised trials. BMJ. 2012;345:e5661.

36. Vargas-Prada S, Serra C, Martínez JM, Ntani G, Delclos GL, Palmer KT, Coggon D, Benavides FG. Psychological and culturally-influenced risk factors for the incidence and persistence of low back pain and associated disability in Spanish workers: findings from the CUPID study. Occup Environ Med. 2013;70:57-62.

37. Occupational health and safety risks in the healthcare sector. Guide to prevention and good practice. Luxembourg: European Union. 2011. file:/// C:/Users/U10052/Downloads/New-EUL14157_Healthcare_Sector_web.pdf. Accessed 10 Sept 2018.

38. Vargas-Prada S, Martínez JM, Coggon D, Delclos G, Benavides FG, Serra C. Health beliefs, low mood, and somatizing tendency: contribution to incidence and persistence of musculoskeletal pain with and without reported disability. Scand J Work Environ Health. 2013;39:589-98.

39. Gadea R, Sevilla M, García A. ERGOPAR 2.0. Un procedimiento de ergonomía participativa para la prevención de trastornos musculoesqueléticos de origen laboral. Madrid: Instituto Sindical de Trabajo, Ambiente y Salud (ISTAS); 2014.

40. Sánchez-López P, Dresch V. The 12-Item General Health Questionnaire (GHQ-12): reliability, external validity and factor structure in the Spanish population. Psicothema. 2008;20-4:839-43.

41. Williams A. EQ-5D concepts and methods. Eds. Kind P, Brooks R, Rabin R. Dordrecht: Springer. 2005:1-17.

42. Schwarzer R, Jerusalem M. Generalized Self-Efficacy scale. In: Weinman J, Wright S, Johnston M, editors. Measures in health psychology: A user's portfolio. Causal and control beliefs. Windsor: NFER-NELSON; 1995. p. 35-7.

43. Bayliss EA, Ellis JL, Steiner JF. Seniors' self-reported multimorbidity captured biopsychosocial factors not incorporated into two other data-based morbidity measures. J Clin Epidemiol. 2009;62-5:550-7.

44. Bishop SR. What do we really know about mindfulness-based stress reduction? Psychosom Med. 2002;64:71-84.

45. García AM, Gadea R, Sevilla MJ, Ronda E. Validación de un cuestionario para identificar daños y exposición a riesgos ergonómicos en el trabajo. Rev Esp Salud Pública. 2011:85:331-40.

46. Ramada JM, Serra C, Amick BC III, Castano JR, Delclos GL. Cross-Cultural Adaptation of the Work Role Functioning Questionnaire to Spanish Spoken in Spain. J Occup Rehabil. 2013;23-4:566-75.

47. Ramada JM, Serra C, Amick BC III, Abma Fl, Castano JR, Pidemunt G, et al. Reliability and Validity of the Work Role Functioning Questionnaire (Spanish Version). J Occup Rehabil. 2014;24-4:640-9.

48. Ramada JM, Delclos GL, Amick BC III, Abma Fl, Pidemunt G, Castano JR, et al. Responsiveness of the Work Role Functioning Questionnaire (Spanish Version) in a General Working Population. J Occup Environ Med. 2014;56-2: 189-94
49. Amick BC III, Lerner D, Rogers WH, Rooney T, Katz JN. A review of healthrelated work outcome measures and their uses, and recommended measures. Spine. 2000;25:3152-60.

50. Organizational Indices Committee of the Occupational Health and Safety Council of Ontario. Benchmarking organizational leading indicators for the prevention and management of injuries and illnesses. Toronto: Institute for Work \& Health; 2011.

51. Linnan L, Steckler A. Process evaluation for public health interventions and research. An overview. San Francisco: Jossey-bass; 2002.

52. Wierenga $D$, Engbers $L H$, van Empelen $P$, Hildebrandt $V H$. The design of a real-time formative evaluation of the implementation process of lifestyle interventions at two worksites using a 7-step strategy (BRAVO@work). BMC Public Health. 2012;12:619.

53. Best ML. An ecology of text: using text retrieval to study a life on the net. Artif Life. 1997:3-4:261-87.

54. Evanoff BA, Bohr PC, Wolf LD. Effects of a participatory ergonomics team among hospital orderlies. Am J Ind Med. 1999:35-4:358-65.

\section{Ready to submit your research? Choose BMC and benefit from:}

- fast, convenient online submission

- thorough peer review by experienced researchers in your field

- rapid publication on acceptance

- support for research data, including large and complex data types

- gold Open Access which fosters wider collaboration and increased citations

- maximum visibility for your research: over $100 \mathrm{M}$ website views per year

At $\mathrm{BMC}$, research is always in progress.

Learn more biomedcentral.com/submissions 